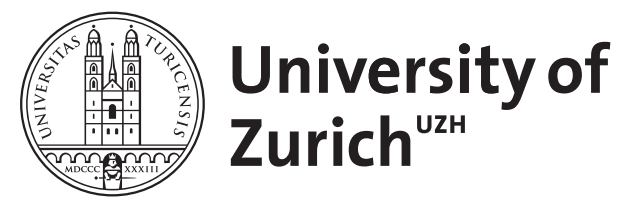

\title{
Rechenstörungen im Kindesalter
}

\author{
von Aster, M ; Kucian, K ; Schweiter, M ; Martin, E
}

\begin{abstract}
Die Prävalenz umschriebener Rechenstörungen ist etwa ebenso hoch wie die der Lese-RechtschreibStörungen. Komorbide psychische Störungen, v.a. Ängste, depressive Symptome und ADHD, sind besonders häufig. Die Entstehungs- und Verlaufsbedingungen sind noch weniger gut erforscht als bei der Legasthenie. Spezifisch Zahlen verarbeitende Hirnfunktionen sind sowohl sprachlich als auch visuellräumlich determiniert und in verschiedenen Hirnregionen lokalisiert. Es kann davon ausgegangen werden, dass bei Kindern mit Rechenstörungen die Prozesse des Aufbaus und der Vernetzung entsprechender neuronaler Strukturen durch Einflüsse aus Anlage und Umwelt behindert werden. Daraus resultieren unterschiedliche Subtypen von mathematischen Lernschwierigkeiten. Individuell angepasste, möglichst schulnahe Therapie- und Fördermaßnahmen müssen auf einer frühzeitigen, inhaltsspezifischen und neuropsychologischen Diagnostik basieren
\end{abstract}

DOI: https://doi.org/10.1007/s00112-005-1166-6

Posted at the Zurich Open Repository and Archive, University of Zurich

ZORA URL: https://doi.org/10.5167/uzh-155797

Journal Article

Published Version

Originally published at:

von Aster, M; Kucian, K; Schweiter, M; Martin, E (2005). Rechenstörungen im Kindesalter. Monatsschrift Kinderheilkunde, 153(7):614-622.

DOI: https://doi.org/10.1007/s00112-005-1166-6 
Monatsschrift Kinderheilkd 2005 • 153:614-622 DOI 10.1007/s00112-005-1166-6

Online publiziert: 22. Juni 2005

(c) Springer Medizin Verlag 2005

Redaktion

M. J. Lentze, Bonn
M. von Aster' ${ }^{1,2}$ - K. Kucian ${ }^{3}$ - M. Schweiter ${ }^{2}$ E. Martin ${ }^{3}$

${ }^{1}$ Klinik für Kinder- und Jugendpsychiatrie, St.-Joseph-Krankenhaus, Berlin

${ }^{2}$ Zentrum für Kinder- und Jugendpsychiatrie, Universität Zürich, Schweiz

${ }^{3}$ MR-Zentrum, Universitätskinderklinik, Zürich, Schweiz

\section{Rechenstörungen im Kindesalter}

\begin{abstract}
Obwohl Rechenstörungen bei Kindern ebenso häufig wie Lese-Rechtschreib-Störungen sind, finden sie bisher in Literatur, Forschung, Diagnostik, Therapie und Förderung nicht in gleichem Maße Beachtung. Hier ist dringend ein Umdenken erforderlich. Entsprechende gesetzliche Regelungen, wie sie schon für Kinder mit Legasthenie existieren, sind zu fordern, die einen Nachteilsausgleich sowie geeignete Förder- und Therapiemaßnahmen gewährleisten.
\end{abstract}

\section{Normale Entwicklung numerischer Kompetenzen}

\section{„triple code model“}

Das von Dehaene [1] formulierte „triple code model“ kann heute als zentraler Bezugspunkt gelten, wenn beschrieben werden soll, wie die geistigen Funktionen der Zahlenverarbeitung und des Rechnens bei erwachsenen Menschen beschaffen sind.

Kurz zusammengefasst besagt es, dass Erwachsene über 3 unterscheidbare, miteinander verbundene neuronale Netzwerke (so genannte Module) verfügen, die entsprechend der verschiedenen repräsentativen Eigenschaften und Funktionen von Zahlen (sprachlich-alphabetisches Zahlwort, visuell-arabische Notation, analoge mentale Zahlenraumvorstellung) in unterschiedlichen Regionen des Gehirns lokalisiert sind und bei umschriebenen Hirnschädigungen zu ganz unterschiedlichen
Teilausfällen führen [2] (• Abb. 1). Dehaene [4] bezeichnet das analoge Modul (den inneren Zahlenstrahl) als Ausdruck eines angeborenen Zahlensinns.

\section{Fähigkeit zur Zahlenverarbeitung in Abhängigkeit vom Alter}

Bei der Frage nach der Entwicklung dieses modularen neurokognitiven Systems für Zahlen ist es unabdingbar, sich auf Ergebnisse der Entwicklungspsychologie und -neuropsychologie zu beziehen. Dabei kommt es sowohl auf die Entwicklung grundlegender, Domänen übergreifender Funktionen wie Intelligenz bzw. Arbeitsgedächtnis an als auch auf die von domänenspezifischen numerischen Funktionen.

Säuglingsalter. Bereits Babys im Alter von wenigen Monaten können Mengen nach ihrer kardinalen Größe erfassen und unterscheiden. Dies wird allgemein als Beleg dafür angesehen, dass es eine angeborene numerische Grundkompetenz, so genannte „core-systems“, [3] gibt, die eine Art Zahlensinn darstellen [4].

Vorschulalter. Die vorschulische Entwicklung ist v. a. dadurch gekennzeichnet, dass Kinder mit Beginn der Sprachentwicklung die Zahlwortsequenz, Zählprinzipien (z. B. Eins-zu-Eins-Zuordnung, stabile Reihenfolge), das $\mathrm{Zu}$ - und Wegzählen zum Verändern von Mengen und Begriffe wie „mehr" und „weniger“ gebrauchen lernen sowie einfache arithmetische Operationen (Additionen und Subtraktionen) durch Zählstrategien auszuführen beginnen.

Die vorschulischen Entwicklungsschritte der Zahlenverarbeitung erfolgen ohne systematische Unterrichtung im Kontakt mit dem sozialen und familiären Umfeld und sind eng an den anschaulichen sensomotorischen Gebrauch der Finger gebunden.

Schulalter. Mit der einsetzenden systematischen Beschulung wird eine 2., nicht linguistische „Zahlensprache“, das arabische Notationssystem, erlernt, welches nur visuell repräsentiert ist und eine ganz eigene, stellenwertbezogene Syntax besitzt, die sich mehr oder weniger stark von den verschiedenen linguistischen Zahlwortsystemen unterscheidet. Die Kinder erlernen und automatisieren damit auch kulturspezifische Übersetzungsregeln für das Übertragen eines gesprochenen oder geschriebenen Zahlworts in die entsprechende arabische Symbolik und umgekehrt. In der deutschen Zahlwortsequenz ergibt sich hier insbesondere die Schwierigkeit der Zehner-Einer-Inversion (einundzwanzig - 21) [18].

Das arabische Notationssystem bildet nicht nur die Grundlage für den Umgang mit größeren Zahlen und den Erwerb von Grundrechenarten und komplexeren Rechenprozeduren, sondern - und dies findet im Schulunterricht weniger explizite Beachtung - auch für den Aufbau einer weiteren Zahlenrepräsentation, nämlich einer inneren abstrakten Zahlenraum- oder Zahlenstrahlvorstellung. Diese ausschließ- 


\section{Hier steht eine Anzeige.}

黛 Springer 


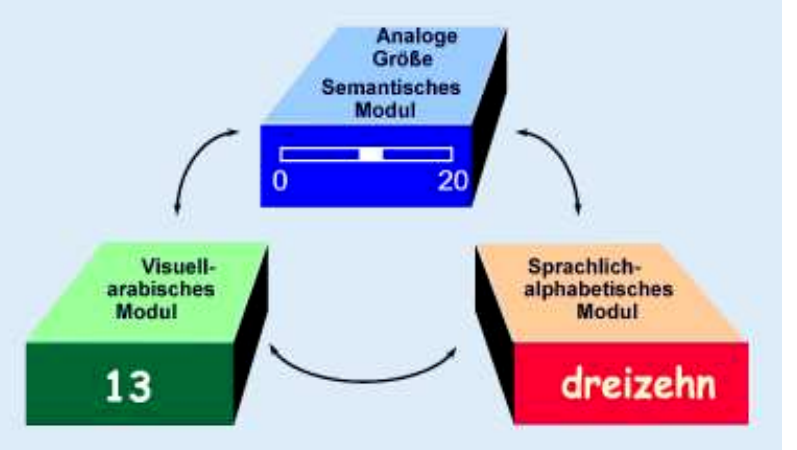

Abb. $1 \varangle$ „triple code model" [1]

lich mentale Repräsentation ordinaler Zahlen ermöglicht es, die Größe einer abstrakten Zahl im Vergleich zu einer anderen $\mathrm{zu}$ bestimmen, sich im Zahlenraum mental zu bewegen und arithmetisch $\mathrm{zu}$ manövrieren, Rechnungen zu schätzen und zu überschlagen.

Das arabische Notationssystem ist für die Entwicklung einer inneren abstrakten Zahlenraum- oder Zahlenstrahlvorstellung notwendig

Untersuchungen an Erwachsenen [5] haben gezeigt, dass Zahlenraum- oder Zahlenstrahlvorstellungen zwar individuell sehr unterschiedliche visuell-räumliche Gestalt annehmen können, aber immer durch arabische Zahlensymbole (nicht etwa durch konkrete Mengen von Objekten) strukturell untergliedert sind und immer mit zunehmender Größe oder Entfernung eine räumliche Kompression erfahren. Das heißt, dass subjektiv der Abstand zwischen 5 und 9 größer erscheint als der zwischen 65 und 69 (Weber-Fechner-Gesetz).

Dass der mentale Zahlenstrahl erst während der ersten Grundschuljahre entsteht, findet einen experimentellen Beleg in der Tatsache, dass der so genannte SNARC-Effekt („spatial numerical association of response codes") erst ab der 2. Klassenstufe nachweisbar ist: Eigenen Untersuchungen zufolge reagieren nur gut $1 / 3$ der Zweitklässler schneller mit der rechten Hand auf größere und schneller mit der linken Hand auf kleinere Zahlen. Bei ihnen existiert also schon eine räumliche, in Schreibrichtung ausgerichtete Vorstellung, die die größeren Zahlen "näher“ an die rechte und die kleineren Zahlen "näher“ an die linke Hand projiziert. Dies ist bei 2/3 der Zweitklässler noch nicht der Fall [6]. Ab der 3. Klasse zeigen Schulkinder dann mehrheitlich, wie Erwachsene, diesen SNARC-Effekt [7].
Zahlen verarbeitende Hirnfunktionen. Ihre Entwicklung ist hierarchisch gegliedert (• Abb. 2) [8]: Die primären Fähigkeiten zur Unterscheidung konkreter (kardinaler) Mengen stellen die bedeutungstragende Grundlage für den Prozess der Symbolisierung dar, und zwar zunächst in Form von Zahlworten und später in Form von schriftlichen Notationssystemen wie den arabischen Zahlen. Der Erwerb des Zahlwortsystems und des arabischen Notationssystems bildet seinerseits die Voraussetzung für die Entwicklung der abstrakt symbolischen, räumlichen (ordinalen) Zahlenraumvorstellung.

Der Prozess der Entwicklung dieser verschiedenen Zahlenrepräsentationen und der an sie geknüpften Funktionen erfolgt einerseits in Abhängigkeit von der wachsenden Kapazität und Verfügbarkeit von Stützfunktionen der allgemeinen Intelligenz (Aufmerksamkeit, Arbeitsgedächtnis, Verarbeitungsgeschwindigkeit) und andererseits erfahrungsabhängig, stellt also einen Prozess dar, der sich in einer individuellen soziokulturellen Umwelt ausprägt, in der diese Funktionen benötigt werden.

Die Entwicklung mathematischer Kompetenz und die Reifung entsprechender Hirnfunktionen vollzieht sich somit auf der Grundlage biologischer Dispositionen im Kontakt mit der sozialen Umwelt und schulischer Instruktion.

Moderne Bild gebende Verfahren wie die funktionelle Magnetresonanztomographie (fMRT) haben es möglich gemacht, die Lokalisation dieser, für numerische Inhalte spezifischen neuronalen Netzwerke zu bestimmen. Dabei sind parietale und präfrontale Gebiete unterschiedlich involviert [2]. Die nonverbalen numerischen Repräsentationen sind in bilateral parietalen Arealen lokalisiert (konkrete Mengen- repräsentation, abstrakte Zahlenraumvorstellung). Im Gegensatz dazu wird numerisches Faktenwissen (Zählen, Ein-malEins) sprachkodiert gespeichert und dementsprechend in links präfrontalen Gehirnarealen verarbeitet. Arabische Zahlen werden durch spezialisierte Gebiete innerhalb des ventralen okzipitotemporalen Pfads für visuelle Identifikation erkannt.

Die Entwicklung dieser neuronalen Netzwerke für Zahlenverarbeitung ist noch kaum untersucht. Erste bildgebende Studien mit Kindern $[9,10,11,12]$ konnten zeigen, dass sie dieselben Regionen im $\mathrm{Ge}$ hirn aktivieren wie Erwachsene.

Die Netzwerke verändern sich plastisch mit zunehmendem Alter und zunehmender Expertise.

So nimmt die Aktivität in den zahlenspezifischen parietalen Regionen zu und in unterstützenden Regionen (anteriorer Gyrus cinguli, verantwortlich für Aufmerksamkeit und Arbeitsgedächtnis) ab (- Abb. 3). Je geübter eine erlernte Funktion ist, desto weniger Arbeitsgedächtnis wird für ihre Ausführung benötigt.

\section{Störungen der Zahlenverarbei- tung und des Rechnens}

\section{Definition}

Im Gegensatz zu den Lese- und Rechtschreibstörungen sind die Entwicklungsstörungen der Zahlenverarbeitung und des Rechnens bislang, was die Mechanismen ihrer Entstehung betrifft, weniger gut erforscht, und zwar obgleich sie, wie man heute weiß, in etwa ebenso häufig sind wie Legasthenien.

- Rechenstörungen werden gemäß der internationalen Klassifikation für psychische Störungen der WHO (ICD-10) als Störungen zentralnervöser Reifungsvorgänge verstanden, die auf verschiedene Weise die Entwicklungsprozesse der kognitiven Informationsverarbeitung behindern.

Die Kriterien, die für eine Diagnosestellung gemäß ICD-10 erfüllt sein müssen oder eine Diagnose ausschließen, gelten analog denen der Lese- und Rechtschreibstörungen. Sie verlangen in erster Linie eine signifikante Diskrepanz zwischen der 
gemessenen und der aufgrund des Alters und der allgemeinen Intelligenz erwarteten Schulleistung in Mathematik, wobei die Anwendung anerkannter standardisierter Tests gefordert wird.

\section{Häufigkeiten}

Untersuchungen in verschiedenen Ländern kommen zu Häufigkeitsangaben zwischen 2 und 6\% [13]. Diesbezügliche Ergebnisse für den deutschsprachigen Raum schwanken zwischen 4,4 und 6,7\% $[14,15,16]$.

- Im Unterschied zu den Lese- und Rechtschreibstörungen scheinen Mädchen eher häufiger betroffen zu sein als Knaben [13, 15].

In einer kürzlich an einer repräsentativen deutsch-schweizerischen Stichprobe durchgeführten Studie, bei der die Kinder zunächst im Vorschulalter und dann am Ende der 2. Klasse untersucht wurden, fanden wir bei insgesamt 6,4\% Leistungen in der Zahlenverarbeitung und im Rechnen (ZAREKI [17]), die um 1,5 Standardabweichungen (SD) unterhalb der Norm lagen (• Tabelle 1). Etwa $1 / 3$ dieser Kinder (2,2\%) zeigten im Lesen und Schreiben (KNUSPEL [18]; SLRT [19]) durchschnittliche Leistungen $(>-0,5 \mathrm{SD})$, bei $2 / 3$ dagegen $(4,2 \%)$ lagen auch die Leistungen in diesem Bereich unterhalb von 1,5 SD (kombinierte Rechen- und Lese-Rechtschreib-Störung). Darüber hinaus zeigte sich, dass der Umfang und das Ausmaß der Defizite bei den Kindern mit kombinierten Störungen deutlich größer waren als bei den Kindern mit umschriebenen Rechenstörungen. Die rechenschwachen Kinder waren überwiegend bereits im Kindergartenalter hinter ihren Altersgenossen zurückgeblieben. Die Geschlechtsunterschiede zeigten sich ebenfalls schon im Kindergartenalter [20]. Am Ende der 2. Klasse waren Mädchen insbesondere bei den umschriebenen Rechenstörungen deutlich häufiger betroffen als Knaben.

Die Untersuchung einer Gruppe von Kindern, die einen Sprachheilkindergarten besuchten zeigte, dass diese ein mehrfaches Risiko für Rechenstörungen tragen.

Monatsschrift Kinderheilkd $2005 \cdot$ 153:614-622

DOI 10.1007/s00112-005-1166-6

(c) Springer Medizin Verlag 2005

\section{M. von Aster · K. Kucian · M. Schweiter · E. Martin}

\section{Rechenstörungen im Kindesalter}

\section{Zusammenfassung}

Die Prävalenz umschriebener Rechenstörungen ist etwa ebenso hoch wie die der Lese-Rechtschreib-Störungen. Komorbide psychische Störungen, v. a. Ängste, depressive Symptome und ADHD, sind besonders häufig. Die Entstehungs- und Verlaufsbedingungen sind noch weniger gut erforscht als bei der Legasthenie. Spezifisch Zahlen verarbeitende Hirnfunktionen sind sowohl sprachlich als auch visuell-räumlich determiniert und in verschiedenen Hirnregionen lokalisiert. Es kann davon ausgegangen werden, dass bei Kindern mit Rechenstörungen die Prozesse des Aufbaus und der Vernetzung entsprechender neuro-

\section{Dyscalculia in children}

\section{Abstract}

The prevalence of dyscalculia is about as high as that of dyslexia. Secondary psychological problems, especially anxiety and depression, are frequently associated symptoms. The circumstances which give rise to this condition and its progression are not as well researched as for dyslexia. Brain functions specialised in number processing are linguistically and visuospatially determined and located in various areas of the brain. It is assumed that the development and the networking of the associat- naler Strukturen durch Einflüsse aus Anlage und Umwelt behindert werden. Daraus resultieren unterschiedliche Subtypen von mathematischen Lernschwierigkeiten. Individuell angepasste, möglichst schulnahe Therapie- und Fördermaßnahmen müssen auf einer frühzeitigen, inhaltsspezifischen und neuropsychologischen Diagnostik basieren.

\section{Schlüsselwörter}

Entwicklung · Zahlenverarbeitung · Neuronale Netzwerke · Dyskalkulie . Prävalenz

ed neural structures are inhibited by genetic and environmental factors. This accounts for various subtypes of mathematical learning difficulties. Therapies and other interventions - individually adapted and closely linked to schools - should be based on early, content-specific and neuropsychological diagnoses.

\section{Keywords}

Development - Number processing . Neural networks - Dyscalculia · Prevalence 


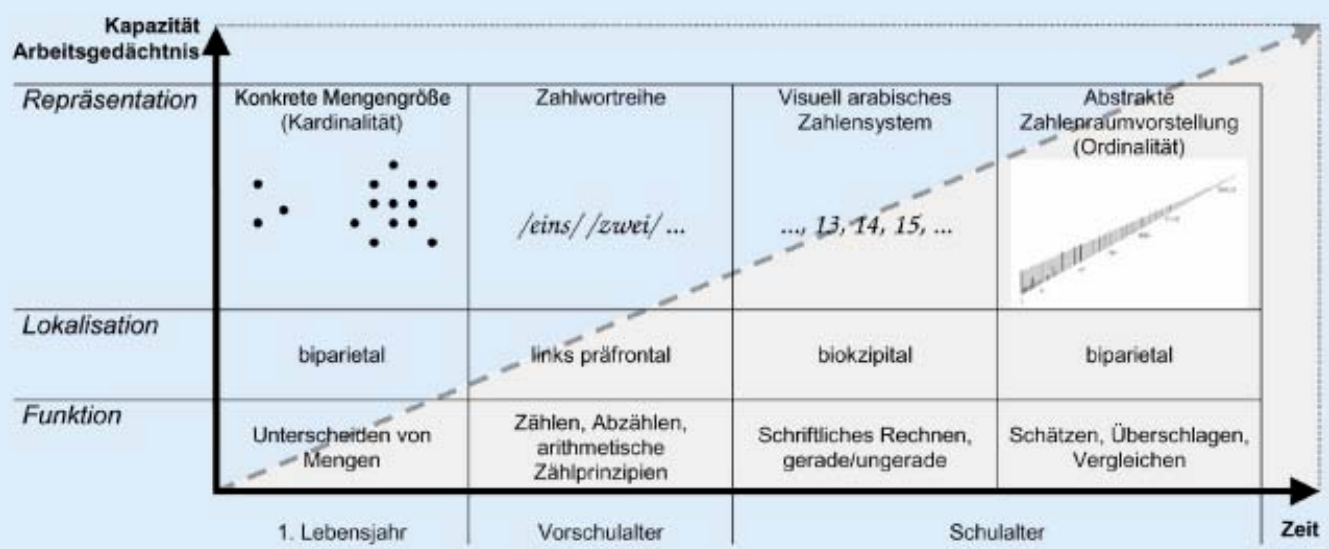

Abb. $2<$ Synopse der Entwicklung Zahlen verarbeitender Hirnfunktionen
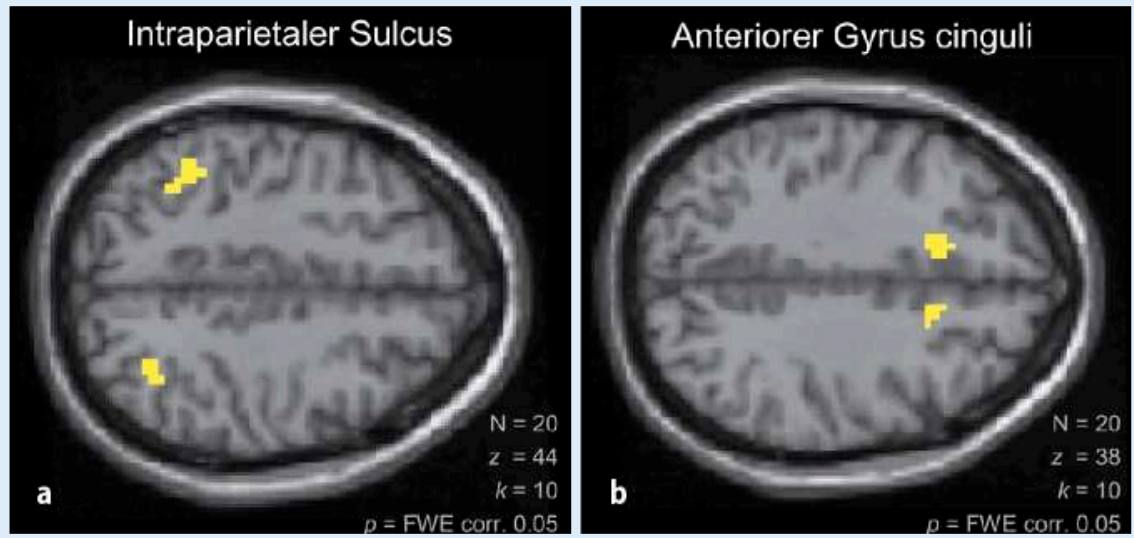

Abb. $3<\mathrm{a}$, b Hirnaktivität bei a Erwachsenen $(27,2 \pm 5,0$ Jahre) und $b$ Kindern $(10,6 \pm 1,5$ Jahre) während approximativem Rechnen (z. B. 2+4 ist eher 15 oder 7), adaptiert aus Kucian et al. 2004 [9]

\section{Langfristiger Verlauf}

Hierzu liegen bislang nur wenig gesicherte Erkenntnisse vor. Ergebnisse einer prospektiv angelegten epidemiologischen Follow-up-Studie aus Israel ergaben, dass bei etwa der Hälfte der Kinder die Kriterien für eine Rechenstörung auch nach 3 Jahren noch erfüllt waren [21].

\section{Psychiatrische Komorbidität}

Es kann davon ausgegangen werden, dass Entwicklungsdyskalkulien im Unterschied zu Legasthenien häufiger mit Ängsten und depressiven Symptomen einhergehen, weniger mit externalisierenden Störungen des Sozialverhaltens [22].

\section{— Häufig entwickeln sich ausgeprägte,} spezifische Mathematikängste, die sich wiederum negativ auf die Leistungsentwicklung auswirken [23].

Etwa 1/3 der rechenschwachen Kinder zeigen Aufmerksamkeits- und Hyperaktivi- tätsstörungen und bei mindestens einem weiteren Drittel bestehen Auffälligkeiten in der Sprachentwicklung, was den relativ hohen Anteil von Kindern erklärt, die sowohl Schwierigkeiten im Schriftspracherwerb als auch beim Rechnenlernen haben [13].

In unserer jüngsten schweizerischen Studie (s. oben) fanden sich insbesondere in der Gruppe der Kinder mit kombinierten Störungen hohe Merkmalsausprägungen für Unaufmerksamkeit und motorische Unruhe.

\section{Klinik}

\section{Syndromale Konzepte}

Die Tatsache, dass die kognitive Symptomatik im Bereich des Rechnens oftmals mit Symptomen in anderen Funktionsbereichen assoziiert ist, hat zur Formulierung verschiedener syndromaler Konzepte geführt. Die bekanntesten stammen von Gerstmann [24] und von Rourke [25, 26].

Schädigungen im Bereich des Gyrus angularis wurden für die 4 Symptome des
Gerstmann-Syndroms, Dyskalkulie, Dysgraphie, Rechts-Links-Orientierungsstörung und Fingeragnosie, verantwortlich gemacht.

Dieses unter Neurologen lange Zeit umstrittene Syndrom (es wurde argumentiert, dass sich diese 4 Symptome auf gleichzeitig bestehende Sprachverarbeitungsstörungen zurückführen lassen; [27]) erfährt im Licht neuerer Ergebnisse mit funktionell bildgebenden Verfahren eine Renaissance, denn diese Ergebnisse belegen, dass die semantische (parietale) Zahlenverarbeitung in unmittelbarer hirnanatomischer Nachbarschaft zu Fingermotorik und visuell-räumlichen Prozessen erfolgt [28]. Kinsbourne [29] beschrieb die Gerstmann-Symptomtetrade erstmals als entwicklungsbezogene Störung bei Kindern („developmental Gerstmann syndrome“, DGS).

Die von Rourke $[25,26]$ in Kanada durchgeführten Untersuchungen über $\mathrm{Zu}$ sammenhänge zwischen schulischen Lernschwierigkeiten und Störungen neuropsychologischer Basisfunktionen führten zur 
Tabelle 1

Prävalenz umschriebener schulischer Entwicklungsstörungen in einer repräsentativen schweizerischen Stichprobe $(n=312)$

\begin{tabular}{lllll} 
CH/ZH & RE [\%] & RE-LRS [\%] & LRS [\%] & Summe [\%] \\
\hline Mädchen & 3,8 & 4,5 & 1,9 & 10,2 \\
\hline Knaben & 0,6 & 3,9 & 1,9 & 6,4 \\
\hline Gesamt & 2,2 & 4,2 & 1,9 & 8,3
\end{tabular}

RE umschriebene Rechenstörung, LRS umschriebene Lese-Rechtschreib-Störung, RE-LRS kombinierte Rechen- und Lese-Rechtschreib-Störung

Definition von 2 unterschiedlichen Syndromen mit Rechenstörungen.

Beim ersten Typ bestanden ausschließlich Lernschwierigkeiten in Mathematik, beim 2. sowohl in Mathematik als auch beim Lesen und Schreiben. Den zuerst genannten Typ bezeichnete Rourke $[25,26]$ als "nonverbal learning disability syndro$m e^{\prime \prime}(N L D)$, den 2. als „subtype RS“ (RS für „,reading" und „spelling“).

Das NLD war insbesondere mit Problemen bei der Durchführung visuell-räumlicher, taktil-kinästhetischer und psychomotorischer Aufgaben assoziiert, zeigte also ein dem DGS ähnliches Muster. Darüber hinaus berichtete Rourke $[25,26]$, dass NLD-Kinder auch häufig internalisierende psychische Störungen entwickeln wie Ängste, Depressionen und Kontaktprobleme. Man fand, dass Kinder mit NLD Schwächen in den nonverbalen Intelligenzfunktionen und Stärken im Bereich der sprachlichen und schriftsprachlichen Funktionen hatten.

Ein genau umgekehrtes Muster von Stärken und Schwächen traf für den „subtyp RS“ zu. Diese Kinder zeigten primär Schwierigkeiten im Bereich der auditivsprachlichen Verarbeitung und Stärken im Bereich der nonverbalen, visuell-räumlichen und psychomotorischen Funktionen. Rourke $[25,26]$ nahm für das NLD ein rechtshemisphärisches und für den Subtyp RS ein linkshemisphärisches Reifungsdefizit an.

Längst nicht alle Kinder, die visuellräumliche Verarbeitungsdefizite, graphomotorische oder Sprachentwicklungsstörungen haben, entwickeln gleichzeitig auch Schwierigkeiten beim Rechnenlernen. Umgekehrt zeigen längst nicht alle Kinder mit Rechenproblemen die Gerstmann-Symptome, visuell-räumliche oder Sprachverarbeitungsstörungen [30].
Die Annahme von Reifungsdefiziten im Bereich neuropsychologischer Basisfunktionen scheint somit zur Erklärung von Teilleistungsstörungen im Rechnen allein nicht ausreichend zu sein (mangelnde Spezifität). Gute visuell-räumliche Fähigkeiten stellen allein ebensowenig eine hinreichende Bedingung für erfolgreiches Rechenlernen dar, wie gute sprachliche oder motorische Funktionen. Dennoch kann angenommen werden, dass diese 3 Bereiche von Grundfunktionen einen wesentlichen Anteil an der Entwicklung und Ausdifferenzierung der spezifischen Zahlen verarbeitenden neuronalen Netzwerkstrukturen haben.

\section{Unterformen}

Für die insbesondere für die Behandlung von Rechenstörungen relevante Frage nach verschiedenen Unterformen (Subtypen) ist die direkte Untersuchung der zahlreichen verschiedenen Aspekte der kognitiven Zahlenverarbeitung und des Rechnens erforderlich.

Mit einem neuropsychologisch begründeten Testverfahren, das verschiedene semantische und kodierungsspezifische Elemente der Zahlenverarbeitung in 12 Subtests erfasst (ZAREKI [17]), konnten 3 Subtypen anhand von Daten rechenschwacher Kinder der 2.-4. Klassenstufe clusteranalytisch unterschieden werden [31]:

- tief greifender Subtyp,

- sprachlicher Subtyp,

- arabischer Subtyp.

Tief greifender Subtyp. Bei den betroffenen Kindern lagen Mittelwertabweichungen von mehr als 1,5 Standardabweichungen in beinahe allen überprüften numerischen Fertigkeitsbereichen vor. Insbeson- dere scheint ihnen ein grundlegendes Verständnis für numerische Größenrelationen und basale Konzepte von „mehr - weniger" und „Teil - Ganzes" zu fehlen.

Nur bei einer Minderheit dieser Kinder bestanden ausschließlich Rechenstörungen. Bei der Mehrzahl fanden sich zahlreiche zusätzliche Entwicklungsauffälligkeiten, insbesondere Lese-RechtschreibProbleme, ADHD und sozial-emotionale Symptome.

Sprachlicher Subtyp. Diese Kindern zeigen signifikante Schwierigkeiten nur beim Kopfrechnen sowie beim Abzählen von Mengen und beim Rückwärtszählen. Die häufigen Fehler beim Abzählen machen bei ihnen auch das zu- und wegzählende Addieren und Subtrahieren fehleranfällig. Dies wiederum erschwert den Aufbau von Abrufstrategien und das Anlegen von Faktenwissen. Die Kinder verharren in unreifen, langsamen Zählstrategien und fallen dadurch schulisch zurück. In dieser Gruppe zeigte etwa die Hälfte der Kinder zusätzliche Symptome eines Aufmerksamkeitsdefizits sowie Rückstände in der Sprachund Schriftsprachentwicklung.

Arabischer Subtyp. Der dritte Subtyp erhielt seinen Namen, weil die betroffenen Kinder in erster Linie Probleme beim Übertragen von Zahlworten in die arabische Kodierung hatten und umgekehrt (arabische Zahlen lesen und nach Diktat schreiben). Diesen Kindern fällt das Erlernen des stellenwertbezogenen arabischen Notationssystems und der entsprechenden Transkodierungsregeln besonders schwer. Überzufällig häufig fanden sich in dieser Gruppe Kinder, die fremd- oder mehrsprachig aufgewachsen sind, und für die die zahlenlinguistischen Besonderheiten der deutschen Zahlwortreihe eine besondere Belastung darstellen [16].

\section{Ätiopathogenese}

Mit Bezug auf die oben dargestellte Synopse zur Entwicklung Zahlen verarbeitender Hirnfunktionen (『Abb. 1) lassen sich Hypothesen über die Entstehung der Rechenstörungen formulieren [32]. Dabei liegt es nahe, ,frühe “Störungen mit weit reichenden Folgen für das Rechnenlernen (im Sinne des tief greifenden Subtyps) von sol- 
chen zu unterscheiden, die im Vorschulund Schulalter entstehen und in ihrer Ausprägung weniger schwer wiegend erscheinen (im Sinne des sprachlichen und arabischen Subtyps). Sowohl bei jenen Störungen mit frühem als auch bei jenen mit späterem Ursprung können der Aufbau und die Automatisierung angemessener Zahlraumvorstellungen erschwert sein, da dieser Prozess auf den verschiedenen inhaltsspezifischen Vorläuferfunktionen („coresystems“, Zählfertigkeiten, arabisches Stellenwertsystem) basiert und auf ausreichende Aufmerksamkeits- und Arbeitsgedächtnisleistungen angewiesen ist.

\section{Früh bedingte Störungen}

Es kann entweder eine genetisch disponierte mangelhafte Ausprägung von Funktionen der Mengenunterscheidung („,Core-systems“) vorliegen und/oder es bestehen frühe Störungen in der allgemeinen Entwicklung von Aufmerksamkeit und Arbeitsgedächtnis, z. B. in Folge frühkindlicher Hirnschädigungen oder auch gravierender Stresserfahrungen. Letzteres stellt nicht nur ein spezifisches (auf die Zahlenverarbeitung bezogenes), sondern auch ein die kognitive und sozial-emotionale Entwicklung allgemein betreffendes Risiko dar.

\section{Später bedingte Störungen}

Die konkrete (kardinale) Mengenrepräsentation und die durch sie gewährleisteten Funktionen sind gut ausgebildet. Probleme ergeben sich aus Störungen im Erwerb der linguistischen und der arabischen Zahlenrepräsentationen, die verschiedene Ursachen haben können.

Die Automatisierung von sprachlich kodiertem Zahlenwissen (Zahlwortsequenz, arithmetisches Faktenwissen) kann durch Einschränkungen in der Sprachentwicklung und/oder Aufmerksamkeitsprobleme behindert werden. Ein zentraler Mechanismus für die erforderlichen Automatisierungsprozesse sind konstante Wiederholungen beim Zählen, Abzählen und beim zählenden Rechnen. Der Übergang von einer Abzähl- zu einer Abrufstrategie setzt voraus, dass das Kind viele Male bei ein und derselben Aufgabe zum selben Ergebnis kommt. Verzählt sich das Kind immer wieder und kommt zu unterschiedlichen Ergebnissen, kann die Assoziationsstärke zwischen Aufgabe und Ergebnis nicht wachsen, und das Kind verharrt in der unreifen Zählstrategie. Solches Verzählen kann z. B. dadurch zustande kommen, dass die Bildung der Zahlworte und die korrekte Sequenzbildung besonders schwer fallen oder dass Kinder sehr flüchtig und ungenau und unter Verletzung der Eins-zu-Eins-Korrespondenzregel zählen.

Bei Kindern die zwei- oder mehrsprachig aufwachsen, entsteht beim Erlernen des arabischen Notationssystems ein besonderes Risiko. Diese Kinder müssen für mehr als ein Zahlwortsystem Übersetzungsregeln ins arabische Notationssystem berücksichtigen. Wenn sie die Übersetzungsregeln der einen Zählsprache auch in der anderen anwenden, kommen ständig Fehlermeldungen, die im schulischen Alltag oft nicht schnell genug erkannt und auf das zugrunde liegende Problem bezogen werden können.

Aber auch andere Besonderheiten in der vorschulischen Lernbiographie können zu sonderbaren und auf den ersten Blick unerklärlichen Abweichungen führen.

\section{- Insbesondere wenn Kinder Zahlen in anderen als numerischen Bezügen gebrauchen, kommt es zu konkurrie- renden Sinnbezügen für Zahlen.}

Ein eindrucksvolles Beispiel liefert die kleine Irma, die in der 2. Klasse von sich behauptet, dass sie nie mehr wird Rechnen lernen können. Die Lehrer sind ratlos und diagnostizieren eine schwere Dyskalkulie:

Irma, ein überdurchschnittlich begabtes und sehr fantasievolles Kind wuchs als Einzelkind zweier Akademikereltern auf. Bereits mit 4 1/2 Jahren begann sie, sich eine Spielgeschichte zu erschaffen, in der die handelnden Personen die Namen von Zahlen erhielten. So erlebte „Drei“, ein blonder und frecher Knabe, mit „Neun", seinem Freund, allerlei Abenteuer, und es bestanden vielerlei Beziehungen zu „Fünf“, „Sechzehn“, "Acht" und vielen Anderen aus der Bullerbü-artigen Nachbarschaft, von deren Biographie und Eigenschaften Irma zahlreiche Einzelheiten zu berichten wusste.

Man kann sich gut vorstellen, in welche Verwirrung Irma geriet, wenn sie 3 von $6 a b$ ziehen sollte.
Auch bei Kindern mit autistischen Störungen beobachtet man bisweilen obsessive Fixierungen auf Zahlen, wobei diese jedoch andere als kardinale, ordinale oder arithmetische Sinnbezüge tragen. Hier werden kalendarische Fakten, Primzahlen, Buslinien, Fahrplanzeiten usw. gesammelt und geordnet. Zahlen haben hier eine eher klassifikatorische, begriffsgebende Funktion, der lexikalische Gebrauch der Zahlen ist daher auch meist völlig ungestört. Der Gebrauch der Zahlen in einem mathematisch-funktionalen Sinnbezug ist jedoch erschwert (vgl. [33]).

Mit Bezug auf die schulische Lernumgebung muss schließlich auf ein weiteres Phänomen eingegangen werden.

Lernen ist nicht nur erfahrungsabhängig (Vorerfahrung und aktueller Lerninhalt), sondern auch abhängig vom momentanen Zustand, in dem sich der Lernende befindet.

Die äußere Lernumgebung und die innere Befindlichkeit beeinflussen diesen Zustand sehr wesentlich. 2 Aspekte verdienen in Hinblick auf das Gelingen schulischer Lernprozesse besondere Erwähnung:

1. Schulische Didaktik: Hier geht es um die Frage, ob curriculare Unterrichtsinhalte und -methoden adaptiv sind im Hinblick auf die Lernziele.

2. Lernen ist abhängig vom inneren Zustand des lernenden Individuums: Hier geht es um die Frage, unter welchen emotionalen und motivationalen Bedingungen Lernprozesse stattfinden und dementsprechend gefördert oder behindert werden.

Hier können Umstände der persönlichen Lebenssituation und daraus resultierende psychische Belastungen ebenso eine Rolle spielen, wie allgemeine Faktoren der schulischen Lernumgebung.

Besonders im Schulalter, aber durchaus auch schon vorher, üben institutionalisierte öffentliche Bewertungssysteme wie die Notengebung einen Selektionsdruck auf die Entwicklung von Fertigkeiten aus. Erfolgreiche Tätigkeiten werden verstärkt und gesucht, erfolglose dagegen gemieden. Bei leistungsschwachen Kindern erzeugt die fortgesetzte Konfrontation mit Misserfolg Stress. Daraus ent- 
stehende Ängste führen zu Blockaden indem sie Aufmerksamkeit und Arbeitsgedächtnis reduzieren und damit weiteren Misserfolg wahrscheinlicher machen. Erwiesenermaßen steigen mit zunehmender Angst auch Bearbeitungszeit und Fehlerraten beim Lösen von Aufgaben. In einem solchen Kreislauf festigt sich auch spezifisches Wissen über die eigene Person („,das kann ich nicht“, „das lerne ich nie“). Es entwickeln sich ungünstige Attributionsstile und Kontrollüberzeugungen.

Das chronische Ausbleiben positiver Handlungs-Ergebnis-Beziehungen führt sukzessive zum Verlust von Motivation und Anstrengungsbereitschaft, Erfolge werden nicht mehr erwartet und Minderwertigkeitsgefühle wachsen.

Die aus der Vermeidung resultierenden Übungsdefizite schwächen schließlich jene neuroplastischen Hirnreifungsprozesse, die für eine erfolgreiche weitere Lernentwicklung notwendig sind.

\section{Diagnostik, Förderung und Behandlung}

Sie sollten grundsätzlich so früh und so integrativ wie möglich erfolgen, d. h. unter Einbeziehung von Lehrern und Eltern.

\section{Diagnostik}

Die störungsspezifische Diagnostik schließt analog den Leitlinien der Deutschen Gesellschaft für Kinder- und Jugendpsychiatrie [34] eine sorgfältige Erfassung der Symptomatik (Schulnoten, Leistungsdiskrepanzen, Fehlermerkmale), der Entwicklungsgeschichte einschließlich relevanter familiärer und schulischer Rahmenbedingungen, der psychischen Befindlichkeit im Allgemeinen und im Hinblick auf die spezifischen Lernschwierigkeiten ebenso ein, wie die Durchführung psychometrischer Testverfahren (Intelligenz, neuropsychologische Basisfunktionen, Zahlenverarbeitung und Rechnen) und eine entwicklungsneurologische Abklärung.

Die neuropsychologische Testbatterie für Zahlenverarbeitung und Rechnen bei Kindern (ZAREKI, [17]) ermöglicht eine inhaltsbezogene Diagnosestellung auf der Grundlage der Überprüfung verschiedener relevanter Aspekte der Zahlenverarbeitung und des Rechnens. Sie gibt damit auch Hinweise auf die Art der Schwie- rigkeiten und die daraus abzuleitenden Ziele von Förder- und Therapiemaßnahmen. Mit der Kindergartenversion (ZAREKI-K) wird demnächst ein Instrument zur Früherkennung von Risiken für eine spätere Dyskalkulie verfügbar sein, das die verschiedenen relevanten Vorläuferfunktionen überprüft [20]. Eine Übersicht über weitere verfügbare diagnostische Instrumente findet sich bei Hasselhorn et al. [35].

\section{Förderung und Therapie}

Sie setzen ein differenziertes befundbasiertes Verständnis der spezifischen Schwierigkeiten des Kindes voraus. Zunächst sollten die Untersuchungsergebnisse den Eltern und Lehrern in jedem Fall sorgfältig vermittelt und erklärt sowie mit Hinweisen für die konkrete Unterrichts- und Hausaufgabengestaltung verbunden werden. Förder- und Therapiemaßnahmen müssen den konkreten Problemen eines jeden Kindes individuell angepasst werden.

Trainings, die sich allein und pauschal auf die Verbesserung der Psychomotorik, der Wahrnehmung oder der Sprache beziehen, können keine ausreichenden Verbesserungen numerischer Kompetenzen bewirken.

Kindern mit schwergradigen oder tief greifenden Rechenstörungen gelingt in der Regel der Aufbau abstrakter Zahlenraum- oder Zahlenstrahlvorstellungen nicht, wodurch das Verständnis arithmetischer Operationen behindert wird. Ihnen muss unter Zuhilfenahme geeigneter Veranschaulichungsmittel geholfen werden, die notwendigen räumlich-numerischen inneren Repräsentationen selbst zu erzeugen. Sofern Schwierigkeiten in der visuell-räumlichen Orientierung, in der Unterscheidung von rechts und links oder in der Graphomotorik bestehen, können ergänzende Übungsbehandlungen für diese Funktionsbereiche indiziert sein, insbesondere wenn sie inhaltsspezifisch erfolgen.

Auch wenn Kinder z. B. infolge von Sprachentwicklungsbesonderheiten oder Aufmerksamkeitsdefiziten Schwierigkeiten haben beim Zählen, Abzählen, Einhalten von Reihenfolgen, Behalten von Zwischenschritten oder Erlernen des arabischen Notationssystems und seiner Regelstruktur, sind inhaltsspezifische Fördermaßnahmen ggf. mit inhaltsübergrei- fenden Unterstützungsangeboten zu kombinieren. Ein empfehlenswertes, wissenschaftlich fundiertes Grundlagenwerk, das auch Hinweise auf eine Vielzahl von bewährten Fördermaterialien enthält, ist das von Lorenz u. Radatz [36] publizierte „Handbuch des Förderns im Mathematikunterricht“.

Standardisierte und in Hinblick auf ihre Wirksamkeit evaluierte Therapieprogramme existieren bislang nicht. Hier besteht erheblicher Nachholbedarf, wenngleich angemerkt werden muss, dass es im Unterschied zu Lese-Rechtschreib-Programmen in der Dyskalkulietherapie häufig zwar auch, aber oft nicht primär darauf ankommt, gewisse Prozesse zu automatisieren, sondern zunächst geeignete innere Repräsentationen und ein Verständnis arithmetischer Algorithmen zu erzeugen. Dies macht ein flexibles und individualisiertes Vorgehen nötig.

\section{O In der Dyskalkulietherapie ist ein flexibles und individuali- siertes Vorgehen nötig}

Förderung sollte, so weit möglich, im Regelunterricht stattfinden können. Dort wo dies nicht praktikabel ist oder nicht ausreicht, sind einzeltherapeutische Fördermaßnahmen bei speziell ausgebildeten Sonder- und Heilpädagogen oder Psychologen sinnvoll. Eine enge Kooperation mit Klassenlehrern ist dabei unbedingt anzustreben.

Begleitende psychotherapeutische, verhaltenstherapeutische und medikamentöse Behandlungsmaßnahmen sind dann einzuleiten, wenn der Schweregrad von Angstsymptomen (Schulangst, Leistungsund Prüfungsangst), depressiven Symptomen oder hyperkinetischen und Aufmerksamkeitsstörungen dies nahelegt.

\section{Fazit für die Praxis}

- Die Fähigkeiten mit Zahlen umzugehen und zu rechnen, entwickeln sich im Vorschul- und Schulalter und gehen mit der neuroplastischen Ausdifferenzierung spezifischer Hirnfunktionen einher.

- Rechenstörungen bei Kindern sind ebenso häufig wie Lese-Rechtschreib- 
Störungen und stellen ein Risiko für die Persönlichkeitsentwicklung dar.

- Häufige begleitende Störungen sind Ängste, Depressionen und ADHD.

- Eine differenzierte neuropsychologische Diagnostik bildet die Grundlage für individuelle Therapie- und Fördermaßnahmen durch ausgebildete Fachkräfte.

- Für Kinder mit Dyskalkulie braucht es dringend gesetzliche Regelungen, wie sie heute analog schon für Kinder mit Legasthenie existieren. Ein Nachteilsausgleich sollte erfolgen und die Finanzierung von Förder- und Therapiemaßnahmen gewährleistet werden.

\section{Korrespondierender Autor PD Dr. M. von Aster}

Klinik für Kinder- und Jugendpsychiarie und Psychotherapie, St.-Joseph-Krankenhaus, Bäumerplan 24, 12101 Berlin

E-Mail: vonaster@kjpd.unizh.ch

Interessenkonflikt: Der korrespondierende Autor versichert, dass keine Verbindungen mit einer Firma, deren Produkt in dem Artikel genannt ist, oder einer Firma, die ein Konkurrenzprodukt vertreibt, bestehen.

\section{Literatur}

1. Dehaene S (1992) Varieties of numerical abilities Cognition 44:1-42

2. Dehaene S, Spelke E, Pinel P et al. (1999) Sources of mathematical thinking: behavioral and brainimaging evidence. Science 284:970-974

3. Feigenson L, Dehaene S, Spelke E (2004) Core systems of number. Trends Cognit Sci 8:307-314

4. Dehaene $S$ (1997) The number sense: how the mind creates mathematics. Oxford University Press, Oxford

5. Seron X, Pesenti M, Noel MP et al. (1992) Images of numbers, or "when 98 is upper left and 6 sky blue". Cognition 44:159-196

6. Schweiter M, Weinhold Zulauf M, Aster M von (2005) Die Entwicklung räumlicher Zahlenrepräsentationen und Rechenfertigkeiten bei Kindern. Z Neuropsychologie (im Druck)

7. Berch DB, Foley EJ, Hill RJ et al. (1999) Extracting parity and magnitude from Arabic numerals: developmental changes in number processing and mental representation. J Exp Child Psychol 74:286308

8. von Aster M (2003) Umschriebene Entwicklungsstörungen des Rechnens. In: Herpertz-Dahlmann B, Resch F, Schulte-Markwort M et al. (Hrsg) Lehrbuch der Entwicklungspsychiatrie. Schattauer, Stuttgart New York

9. Kucian K, Loenneker T, Dietrich T et al. (2004) Children's brains learn approximate calculation. In: Rem schmidt $\mathrm{H}$, Belfer M (eds) Book of Abstracts of the 16th World Congress of the International Association for Child and Adolescent Psychiatry and Allied Professions (IACAPAP). Steinkopff, Darmstadt
10. Rivera SM, Reiss AL, Eckert MA et al. (2005) Developmental changes in mental arithmetic: evidence for increased functional specialization in the left inferior parietal cortex. Cereb Cortex 16

11. Qin Y, Carter CS, Silk EM et al. (2004) The change of the brain activation patterns as children learn algebra equation solving. Proc Natl Acad Sci USA 101:5686-5691

12. Temple E, Posner MI (1998) Brain mechanisms of quantity are similar in 5-year-old children and adults. Proc Natl Acad Sci USA 95:7836-7841

13. Shalev RS, Auerbach J, Manor O et al. (2000) Developmental dyscalculia: prevalence and prognosis. Eur Child Adolesc Psychiatry [Suppl 2] 9:11581164

14. Hein J, Bzufka N, Neumarker K (2000) The specific disorder of arithmetic skills. Prevalence studies in a rural and an urban population sample and their clinico-neuropsychological validation. Z Entwicklungspsychol Pädagog Psychol [Suppl 2] 9:87-101

15. Klauer K (1992) In Mathematik mehr leistungs schwache Mädchen, im Rechnen und Rechtschreiben mehr leistungsschwache Jungen? Zur Diagnostik von Teilleistungsschwächen. Z Entwicklungspsychol Pädagog Psychol 24:48-65

16. Aster MG von, Deloche G, Dellatolas G et al. (1997) Zahlenverarbeitung und Rechnen bei Schulkindern der 2. und 3. Klassenstufe: Eine vergleichende Studie französischsprachiger und deutschsprachiger Kinder. Z Entwicklungspsychol Pädagog Psychol 29:151-166

17. Aster M von (2001) ZAREKI (neuropsychological test battery for number processing and calculation in children). Swets \& Zeitlinger, Lisse

18. Marx H (1998) Knuspels Leseaufgaben. Gruppenlesetest für Kinder Ende des ersten bis vierten Schuljahres. Hogrefe, Göttingen

19. Landerl K, Wimmer H, Moser E (1997) Salzburger Lese- und Rechtschreibtest. Verfahren zur Differentialdiagnose von Störungen des Lesens und Schreibens für die 1. bis 4. Schulstufe. Huber, Bern Stuttgart Toronto

20. Weinhold Zulauf M, Schweiter M, Aster MG von (2003) Das Kindergartenalter: Sensitive Periode für die Entwicklung numerischer Fertigkeiten. Kindheit Entwicklung 12:222-230

21. Shalev RS, Manor O, Auerbach J et al. (1998) Persistence of developmental dyscalculia: what counts? Results from a 3-year prospective follow-up study. J Pediatr 133:358-362

22. Aster MG von (1996) Psychopathologische Risiken bei Kindern mit umschriebenen schulischen Entwicklungsstörungen. Kindheit Entwicklung 5:5359

23. Ashcraft M, Faust M (1994) Mathematics anxiety and mental arithmetic performance: an exploratory investigation. Cognit Emot 8:97-125

24. Gerstmann J (1930) Zur Symptomatologie der Hirnläsionen im Übergangsgebiet der unteren Parietal- und mittleren Occipitalwindung. Nervenarzt 3:691-695

25. Rourke B (1993) Arithmetic disabilities, specific and otherwise: a neuropsychological perspective. J Learn Disabil 26:214-226

26. Rourke B (1989) Nonverbal learning disabilities. The syndrome and the model. Guilford Press, New York

27. Poeck K, Orgass B (1975) Gerstmann syndrome without aphasia: comments on the paper by Strub and Geschwind. Cortex 11:291-295

28. Butterworth B (1999) The mathematical brain. Macmillan, London

29. Kinsbourne M (1968) Developmental Gerstmann syndrome. Pediatr Clin North Am 15:771-778
30. Aster MG von (1994) Developmental dyscalculia in children: review of the literature and clinical validation. Acta Paedopsychiatr 56:169-178

31. Aster MG von (2000) Developmental cognitive neuropsychology of number processing and calculation: varieties of developmental dyscalculia. Eur Child Adolesc Psychiatry 9:41-57

32. Aster MG von (2005) Wie kommen Zahlen in den Kopf? Ein neurowissenschaftliches Modell der Entwicklung zahlenverarbeitender Hirnfunktionen. In: Aster MG von, Lorenz JH (Hrsg) Rechenstörungen bei Kindern. Neurowissenschaft, Psychologie, Pädagogik. Vandenhoeck \& Rupprecht, Göttingen

33. Aster MG von, Zachmann M, Brandeis D et al. (1997) Psychiatric, neuropediatric and neuropsychological symptoms in a case of Hypomelanosis of Ito. European Child and Adolescent Psychiatry 6:227-233

34. Deutsche Gesellschaft für Kinder- und Jugendpsychiatrie und Psychotherapie (2000) Leitlinien zu Diagnostik und Therapie von psychischen Störungen im Säuglings-, Kindes- und Jugendalter. Deutscher Ärzteverlag, Köln

35. Hasselhorn M, Marx H, Schneider W (2005) Diagnostik von Mathematikleistungen. Hogrefe, Göttingen

36. Lorenz J, Radatz K (1993) Handbuch des Förderns in Mathematikunterricht. Schroedel, Hannover

\section{Sie suchen eine neue Stelle?}

Warum sich lange nach passenden Angeboten umschauen? Vielfältige Jobangebote gibt es online unter www.jobcenter-medizin.de Die Vorteile:

- nutzerfreundliches, übersichtliches Design

- schnelle, komfortable, präzise Suchfunktionen

- Erstellung persönlicher Suchprofile und Benachrichtigung über aktuelle, offene Angebote per Mail fachspezifische Stellensuche 